\title{
Evaluating Life Events and Chronic Stressors in Relation to Health: Stressors and Health in Clinical Work
}

\author{
Töres Theorell \\ Institute for Stress Research, Stockholm University, Stockholm, Sweden
}

\begin{abstract}
Despite the fact that there is an extensive scientific literature regarding the importance of exposure to psychosocial stressors, the assessment of such stressors is often neglected in clinical work. The present review summarizes the scientific literature on critical life changes and work-related stressors. Particular emphasis has been on somatic outcomes and physiological processes that have been shown to be affected by exposure to stressors. Although the relationships are highly complex, it could be concluded that exposure to stressors may often determine the onset of many illnesses. Standardized well-functioning assessment instruments that could be used in clinical practice exist and should be used.

Copyright ๔ 2012 S. Karger AG, Basel
\end{abstract}

The interplay between the individual characteristics of a patient and the situation surrounding him/her determines the effects of stressors on the patient's health. This has led to the misinterpretation that the nature and intensity of stressors could be disregarded because the only thing that really counts is the individual reaction. It is enough to point out for instance that deaths related to the loss of a loved person would not occur if loved persons would never die. Death of a close relative does not affect all individuals in the same way. That, however, does not mean that the event per se is unimportant. Critical life events may have a decisive role in determining the onset of a disease [see for instance 1].

An external condition that demands excessive adaptation from an individual is 'filtered' through the individual coping program. This is constituted by genetic factors and previous experiences [see 2]. The interaction between stressor and individual coping program determines the behavioral, psychological and physiological response to the stressor. One of the possible responses is a general arousal reaction. If this arousal reaction lasts for a long time, the response will gradually change. In Hans 
Selye's terminology, this is the general adaptation syndrome that has three stages, arousal, defense and exhaustion. According to McEwen [3], long-lasting adaptation to high arousal leads to allostasis (for a more detailed discussion, see below).

Throughout life, there is a constant flow of experiences. This means that the individual program is never 'ready', it is going through changes throughout life.

The aim of this chapter is to provide a theoretical framework for the assessment of life events and chronic stressors and to review the literature supporting or refuting the influence of stressors on illness development. A second aim is to propose assessment tools that could be used in clinical practice. Since the psychosomatic approach relates psychosocial processes to physiology and somatic illness, the examples that I choose will have 'objective' outcomes, for instance myocardial infarction (which has a defined onset in time and therefore is more accessible for studies of time relationships between stressor and illness) or physiological repeated outcome measures whenever this is possible.

\section{Stressors}

Stressors are always divided into critical events with a defined onset and duration on one hand and chronic stressors, or chronic life difficulties [1] with undetermined onset and duration, on the other hand. Chronic life difficulties are typically conditions that last for a lifetime or for very long periods such as life-long poverty and unresolved marital discord. The border may not be easy to define in all cases however. For instance, a natural disaster such as a tsunami, an earthquake or a nuclear power station emergency are examples of disasters that may result in chronic difficulties such as physical handicap, posttraumatic stress disorder or in a series of secondary events (loss of residence, loss of relatives, loss of job opportunities, etc). An effort to disentangle chronic life difficulties from critical life events has been made by Brown et al. [1].

\section{Critical Life Events}

There are two ways of recording critical life events.

First of all, subjects who go through a defined life event such as job loss, bereavement or reorganization at work could be studied separately. In such studies, the ideal is to follow different phases before, during and after the event. There are numerous such studies that have been published. For instance, longitudinal studies of subjects going through job loss [4, 5], bereavement [6] and hurricanes [7] have been published that relate the different phases of these critical life changes to the development of mental symptoms and physiological reactions. This is of relevance for clinicians who frequently encounter patients that go through such crisis situations but also for public health officers in charge of prevention programs. 
Secondly, critical life change in general can be studied as they occur spontaneously in the flow of people's life. This is particularly relevant for practitioners who could use clinical interview techniques for all of their patients. Two quite different assessment theories have been established, one of them based upon self-administered questionnaires and the other one on systematic interviews.

\section{Standardized Methods for Recording Typical Critical Life Events That People May Experience}

In the most frequently used methodology for recording critical life events, subjects are confronted with lists of critical life events and asked to report whether they have experienced any of them during a defined period of time retrospectively. Such questionnaires were introduced by Holmes and Rahe [8]. The lists have been refined and put into theoretical context. For each change, a score has been assigned based upon population studies. This score indicates mean level of adaptation that the average subject in the population would require for coping with the change. For the time interval studied, these scores are added. For instance, if a person has lost his/her job and divorced during the past 6 months, the population adaptation score for job loss and the corresponding score for divorce are added for this time interval and a total life change unit sum is achieved [for further introduction and theoretical discussion see Rahe 9]. Even short time intervals were used for the study of weekly life events in relation to physiological change. In a study of male patients who were back to work after a myocardial infarction, Theorell et al. [10] showed that a change in weekly life change sum from one week to the next (calculated on the basis of repeated weekly standardized interviews) explained statistically $10 \%$ of the concomitant change in urinary output of adrenaline during work hours in this kind of clinical sample. It should be pointed out however that there was a pronounced interindividual variance in strength of association; for one third of the subjects no relationship was found. Prominent events in this study were conflicts at work and at home, changes in work hours or conditions and personal outstanding success.

More recent research has shown that although the life change unit sums are helpful in predictions of ill health, they are highly correlated with number of critical life events, and the theoretical rationale for additivity has therefore been questioned. New lists of critical life changes have been renewed and validated $[11,12]$. In more recent studies of objective health outcomes such as myocardial infarction, shorter lists of high impact life events are usually used and summing of weights has been abandoned. Weighting is still used but rather in the form of self-rated impact ratings from the participants themselves than from the population.

An example of more recent life change lists utilized in a study of myocardial infarc-

cos s tion patients is from Möller et al. [13] (see table 1 in online suppl. appendix). In this Sty study (the so called SHEEP, Stockholm HEart Epidemiology Programme), all cases of 
myocardial infarction were contacted within a defined geographical area during a defined time period. At the same time, matched population controls were contacted. There were 1,000 men and 500 women in each group. Weight sums of life changes during the 12 months preceding myocardial infarction (and the corresponding period) did not differentiate the groups. However, a crude rating was performed by each subject (for instance conflict 'affected me strongly/fairly/not much'), and this rating turned out to be important. 'Impaired economic situation', 'conflict at work' and 'increased responsibility at work' - if they were reported to 'affect fairly or very strongly' - differentiated clearly both male and female myocardial infarction cases from controls. A relevant critique against this kind of retrospective case control study is that retrospective 'search for meaning' could have influenced the findings. The findings are however consistent also with a prospective study in which healthy men were followed during a one-year period with regard to incidence of myocardial infarction [14]. The life event 'increased responsibility at work during the past 12 months' predicted increased risk of myocardial infarction but a weighted life event sum score of life changes in general was not associated with increased myocardial infarction risk. These findings are also consistent with another large case-control study of myocardial infarction patients [15] in which a standardized interview was used (see below).

A methodological novelty in the SHEEP study was the co-called ONSET interview analysis which focuses on triggering events. The interview goes into detail with regard to the $24 \mathrm{~h}$ preceding the myocardial infarction and possible triggering events occurring during this short period. A similar analysis was also done for the week preceding the onset. These analyses were confined to the cases. They showed that a high pressure deadline' at work was statistically associated with the 24-hour triggering and 'event where felt pressure or competition' as well as 'praised by the boss' were statistical 'triggers during the past week'. We can possibly see parallels with the findings in the weekly life change study described above in which work changes and conflicts as well as outstanding success were prominent features. 'Praise by the boss' could in fact be interpreted as an effort from the boss to increase pressure and competition. There has been a scientific discussion regarding the reliability of these kinds of retrospective short-term trigger studies. However, for the kinds of events that were reported in the SHEEP study the findings are probably reliable, although the magnitude of the association may have been inflated [16].

\section{Triggers and Habitual Levels of Critical Life Changes}

The trigger perspective is related to another theoretical perspective in this research. In early retrospective research on life events preceding illness onset the hypothesis tested was that an excessive accumulation of critical life changes during a short period markedly exceeding the person's habitual level of changes would increase risk of illness. For instance, retrospective studies of 3-month intervals (using summation of 
life change units) showed a pronounced accumulation of change during the months before the onset of myocardial infarction compared to the preceding months [for a summary regarding the accumulation theory see Rahe 9]. Similarly patients who have attempted suicide but survived report a strong accumulation of life events during the month preceding the attempt [17]. Such studies can be criticized because the patients or their relatives may have been 'seeking meaning' retrospectively.

Regardless of interpretation, it is certainly an important observation that subjects could be accustomed to a high or a low incidence of life change in general. Those who become accustomed to a high incidence of changes may belong to privileged intellectually and socially stimulated groups with a low general incidence of illness. Or vice versa, they could belong to a disadvantaged group which is exposed to a high incidence of markedly negative events. An epidemiological study [18] of 28-year-old men (followed up 10 years after they had gone through mandatory medical screening for medical service) showed that participants with high blood pressure at rest reported fewer life events during the past year than did those with normal blood pressure. Such findings illustrate, consistent with previous reasoning that the effect of a large number of events during a given time period should be related to the habitual level of life events experienced by the individual. Regardless of where the scientific discussion will end, it is important to take the general dynamics of change patterns into account with the patient. In addition, the clinical impression is that patients who have just had an onset of a serious illness benefit from discussing critical life changes preceding it.

\section{Positive and Negative Life Changes}

An additional important discussion in life event research relates to positive and negative impact, respectively. The original theory postulates that an accumulation of critical life changes, regardless of whether they have a positive or a negative impact, could trigger illness due to the amount of adaptation demanded. The applicability of this theory probably varies with the kind of illness studied. In patients with coronary heart disease who suffer from attacks of myocardial ischemia whenever excessive physical effort or psychological arousal arises, positive or negative impact may not be so important for the timing of an attack of angina pectoris. This is exactly what we observed in the longitudinal study of myocardial infarction patients described above [10] - angina pectoris attacks could be triggered by positive as well as by negative events. However, for more long-lasting pathophysiological processes, negative impact is more important than positive. A small longitudinal study [19] of subjects who were followed with regard to spontaneously occurring life change on four occasions during a year showed that life changes with negative impact (according to the subjects themselves) were associated with rising levels of plasma prolactin concentration as well as rising serum triglycerides and blood pressure. Positively loaded life changes on the other hand were associated with decreasing serum triglycerides and blood pressure. A psychosomatic 
study was performed on 18-year-old men who were grouped - during the mandatory medical screening procedure for military service - into hypertensive, normotensive and hypotensive subjects. There was a significant difference between the groups [20]. A retrospective life change interview showed that the hypertensive subjects reported that fewer positive events had occurred during their teenage years than did subjects in the other groups. This may be an illustration of the potential importance of a low number of positive life changes during childhood. The positive/negative argument could also be applied to an event's desirability [17] and controllability [21] and also whether it represents an exit or an entrance in the person's life [22]. Such aspects have also been studied by means of interviews and questionnaires.

Later developments of this research tradition has resulted in a comprehensive questionnaire including not only questions about life events, working conditions and social support but also about individual coping patterns [23; see also www.drrahe. com]. Particularly when such questionnaires are used longitudinally, they offer valuable clinical insight.

\section{Detailed Interviews Regarding Critical Life Events - A Sociological Perspective}

A group of researchers was very critical of the questionnaire-based life change inventories. They claimed that self-administered questionnaire surveys can never establish [see 1, 24 and 25] causality since there has to be more rigorous interview data about timing, meaning and context of the reported life changes. They claimed that it would be possible by means of a detailed interview to establish whether the 'event' described should be labeled a 'life event' or a 'chronic life difficulty'. The interview should also be able to examine timing of the event, degree of contextual threat (the most important events were labeled 'markedly threatening'), etc. Whether to label an event 'markedly threatening' or not should always be a matter of context. For instance, a pregnancy is under most circumstances not threatening but it could be a markedly threatening event for a young girl who lacks social and family support. A particularly important argument for using interview rather than questionnaire is that it is only in the interview that it is possible to disentangle what came first, critical life change or illness.

On the basis of their methodological considerations, this group has constructed an interview guide. It has been used particularly for the study of life events in relation to the onset of depression [24], but more recently also for the study of traumatic life events during childhood [25]. According to these researchers, the systematic interview Life Events Diagnostic Survey provides reliable estimates of critical life changes that have occurred a long time before the interview. This interview technique has a well-designed format, and the only disadvantage is that the systematic interrogation about life events that the patient has experienced may take a long time.

A particularly interesting concept that has been introduced by this group is the 'brought forward time' corresponding to the time it may take on average from the 
Table 1. Psychosocial work environment factors of relevance for health of employees

Excessive demands (qualitative as well as quantitative)

Lack of decision latitude

Lack of social support from superiors and/or work mates

Low rewards (material as well as sociological and psychological)

event to the onset of the illness. In the comparison between depression and psychotic schizophrenic outbreak, the brought forward time for depressive episode is typically much longer than it is for an episode of schizophrenia [24].

Approximately at the same time as the Brown group started developing their interview guide, Paykel and his group also started establishing an interview guide which is now named the Interview for Recent Life Events. This interview guide is based upon principles similar to those developed by Brown and Harris [24] but is less detailed. The interview guide contains 64 different life events. There are short specific criteria for some of the events, such as 'serious argument' which is defined as a one-way or interactive altercation which adversely affects behavior of one of both parties for a minimum of 5 days. The interview guide has been used for the exploration of critical life events in relation to onset of several kinds of illnesses such as myocardial infarcCo STM tion and depression [24] (see table 2 in online suppl. appendix).

\section{Work and Unemployment as Stressors}

That adverse job conditions can have strong effects on risk of developing serious illness such as myocardial infarction and depression has been shown in extensive research [26-28]. That this is a cause of financial losses in working life has been shown in several publications [27, 29]. A list of psychosocial conditions that increase health risks is presented in table 1 .

The combination of excessive psychological demands and low decision latitude has been labeled job strain. Several prospective studies have shown that this is a risk factor for myocardial infarction in men [30-32], although there is still disagreement regarding the effect of adjustment for accepted cardiovascular risk factors such as tobacco smoking, high blood pressure and abnormal serum lipids and social class. This means that some authors are of the opinion that there is a direct psycho-neuroendocrinological effect of stress which explains the association, whereas others claim that the association is mediated or confounded by more established risk factors including social class. There is no doubt, however, that psychosocial work environment factors have direct pathophysiological effects. The question is of course how important these effects may be in relation to other relevant pathophysiological processes. The 
'stress' effects are added on vulnerability factors. The estimation of the contribution of the psychosocial factors is complicated by the fact that different mechanisms have different time frames. In the estimation of the role of psychosocial factors in work compensation cases, it may be impossible to really know what the effect of the psychosocial work stressors may have been in an individual case although there is sufficiently strong epidemiological evidence. What pathophysiological mechanisms have been discussed?

Effects of psychosocial work stressors on immunological parameters [33] and on blood pressure regulation [34] have been discussed as mediating mechanisms. A longitudinal study by our group has shown that at least in men increasing job strain is associated with increasing sleep disturbance and blood pressure [35] as well as decreasing plasma testosterone [36] and immunoglobulin G concentration [37]. Decreasing decision latitude has also been associated with decreasing heart rate variability, which indicates a lowered parasympathetic and/or elevated sympathetic activity [38]. Similar observations have been observed for the combination of high effort and low reward, although the physiological correlates of the effort-reward and demand-control-support models may be differently patterned [39]. The evidence regarding the relationship between the psychosocial work environment variables listed above and physiological parameters has been summarized recently by Hansen et al. [40]. When work conditions deteriorate, catabolic variables (associated with energy consumption in situations requiring energy) show increasing activity and anabolic/ regenerative variables (associated with building of resources) decreasing activity.

When the demand control support model was introduced during the 1980s, most studies showed that decision latitude was the most important component. During later years, however, the psychological demands component seems to have become more important, possibly reflecting markedly increasing demands in working life in the (mostly highly industrialized) populations studied [41]. According to summaries, high job strain in men (in most studies the $20-25 \%$ with the worst combination of scores) assessed by means of self-administered questionnaires is associated with a $40 \%$ excess risk of developing a myocardial infarction during 5-10 years of followup after adjustment for other risk factors. Men below 55 years of age at start show a stronger excess risk - in the order of $80 \%$. Among women, the combination of family stress and job stress seems to be more important [42], and predictions of risk with the use of job strain assessment alone are less successful. There are similar observations for the effort reward model. The crucial element in this theory is 'non-reciprocity or imbalance between efforts spent and rewards received'. Not as many prospective studies have been published on the relationship between effort reward and risk of myocardial infarction, although the evidence is pointing at a strong relationship [32]. With regard to depression, both models have shown predictive value in several prospective studies [43]. It has been emphasized by several authors that the two models are complementary, if both are used together better predictions are obtained with regard to health outcomes than if either one is used. 
A third model that has been introduced in work environment research is the 'organizational justice' model which postulates that an organization which is perceived as not providing employees with possibilities to get a fair treatment in crisis situations will generate an increased incidence of both somatic and psychiatric illness. There are few published prospective studies, but there is evidence for an association with myocardial infarction risk [31, 32].

\section{How to Measure the Psychosocial Work Environment?}

As with life changes, the best way of examining a patient's work environment is always by means of a personal interview combined with observations in the work place. However, very good questionnaires have been developed which incorporate the dimensions discussed above. Several of these questionnaires have abbreviated forms that are useful in diagnosing the patient's working conditions. The most comprehensive questionnaire covering most of the aspects discussed above is the Danish Copenhagen Psychosocial Questionnaire (COPSOQ) which has been translated into several languages [44]. COPSOQ has three versions, one short version for screening purposes, one for occupational clinical analysis and one for detailed research purposes. The questionnaire most extensively used for the assessment of the demand control support model is the Job Content Questionnaire (JCQ; Karasek RA, user guide http://html-pdf-convert.com/cari/job-content-questionnaire-user-guide-karasek.html). A shorter version [34] which is similar to the JCQ has been used mostly in Scandinavia (DCQ, 35). This version has a smaller number of questions related to decision latitude and a different response format (based upon frequency rather than intensity). The social support dimension differs from the corresponding one in JCQ. The standardized questionnaire version of Effort Reward Imbalance assessment has been presented by Siegrist et al. [45].

The benefit of examining and intervening in work environments has recently been illustrated by evaluations of manager education programs aiming at improved psychosocial competence among managers which could beneficially influence the work environment $[46,47]$.

The importance of a poor psychosocial work environment has recently been discussed [48] in relation to the concept 'posttraumatic embitterment syndrome' which has been defined by Sensky as:

'an emotion encompassing persistent feelings of being let down, insulted or being a loser, and of being revengeful but helpless. According to the diagnostic criteria of the syndrome, people experience embitterment in response to a single exceptional negative life event which is regarded as the cause of embitterment. Those experiencing embitterment report repeated intrusive memories of the critical event and blame themselves for the event, for not having prevented it or for not being able to cope with it'. 
Sensky argues that the single event criterion may not be valid and that instead 'stress of conscience' (collision between ethical standards that for instance health care staff have been taught on one hand and increasing lack of resources and time making it impossible to meet such standards on the other hand) as well as 'organizational injustice' are key factors. He also argues that chronic embitterment may frequently result in allostatic overload. The discussion regarding chronic embitterment clearly illustrates the problems that researchers in this field are facing in efforts to disentangle the effects of chronic stressors from those of critical life events.

\section{Unemployment}

Unemployment is a powerful stressor in most populations. There has been an extensive discussion regarding the relationship between unemployment and poor health: Have unemployed subjects poorer health to start with or does exposure to unemployment contribute to poor health? Both explanations seem to be valid [for a more detailed discussion see 49]. Exposure to unemployment is therefore an important clinically relevant factor. The effect of unemployment is different across different social and national groups, however. In countries without unemployment insurance systems and with poverty, the health consequences are more severe than in other countries [see for instance 50], and within countries with such systems those with more financial insecurity are likely to suffer more deterioration of health than others [51]. In addition, the effects vary according to phase of unemployment. This has been described in two longitudinal studies $[4,5]$. These show that anxiety levels tend to be particularly high during the anticipation phase (when subjects have been forewarned about upcoming redundancies) and also when several weeks have passed without new employment. During the weeks immediately following job loss, however, the anxiety levels are lower. These fluctuations in affective states are paralleled by changes in physiological parameters such as urinary excretion of catecholamines, blood pressure and serum concentration of serum lipids and cortisol. Elevated risk of severe health consequences such as suicide and myocardial infarction tends to arise when several months of unemployment have passed. This is also the period when more long-lasting consequences for the immune system have been observed [52]. Longlasting unemployment in youth seems to be particularly dangerous because it may have long-term consequences on health-related life habits and on health [53].

\section{Combinations of Social Background/Childhood Adversity and Adult Life Stressors}

Several recent studies have shown that an accumulation of critical life events and/or chronic difficulties during childhood may increase the vulnerability to adult life stressors. For instance, in a recent study, our group has shown that 'adversity in childhood' 
(operationalized as an index comprising residential mobility and crowding, parental loss, parental unemployment and parental physical and mental illness including substance abuse) interacts with job strain in generating allostatic overload, a combined measure of chronic stress (see below). Those who had had increased adversity in childhood were more likely when they were reporting job strain to show a high allostatic load (AL) in younger middle age. Those without childhood adversity, on the other hand, were statistically unaffected by job strain with regard to AL [54].

\section{Physiological Correlates}

There are many physiological parameters that could be studied in relation to stressors. It should be emphasized that, for the clinician, assessment of physiological variables is particularly meaningful when a patient is followed on several occasions so that development over time could be recorded. Measurement of single variables such as salivary cortisol at one point of time is mostly not meaningful. However, composite variables including several physiological variables could be valuable and definitely of prognostic significance in clinical work. One concept that has been used is AL.

Allostatic overload $[3,55]$ corresponds to the physiological result of accumulated 'wear and tear' resulting from the accumulation of stressful exposures. In the short run, such exposures activate physiological stress systems (e.g. the hypothalamicpituitary-adrenal axis) which lead to compensatory changes in secondary physiological systems [e.g. metabolic systems, 56]. These dynamic responses are necessary for the maintenance of homeostasis, a process called allostasis. However, the model of AL suggests that in the long run the accumulation of adaptations mediated by stress systems develops into allostatic overload, a multi-systemic pre-disease state represented by sub-clinical levels of metabolic immunological, endocrine and cardiovascular parameters. AL has been shown to be a useful concept for predicting future morbidity, mortality and psychosocial functioning [57-59]. An AL measure typically includes systolic and diastolic blood pressure, body mass index, waist circumference as well as levels of serum cholesterol, high-density lipoprotein, triglycerides, apolipoprotein $A_{1}$, apolipoprotein $B$, glucose, C-reactive protein and diurnal cortisol. The different domains blood pressure, overweight, atherogenic lipids, protective lipids, immunological status and HPA axis activity (mostly cortisol in plasma or saliva) should be equally represented in the total measure. The clinician could also use proxy measures which include only part of these measures as long as the different dimensions are represented.

The metabolic syndrome is a concept which is close to $\mathrm{AL}$, with a similar set of variables. Chandola et al. [60] have shown in a large longitudinal cohort study (Whitehall II, British civil servants) that repeated exposure for many years to lack of social support at work in combination with job strain ('iso strain') is associated with increased risk of developing metabolic syndrome. 
In clinical work, it is important to remember that there is substantial evidence indicating that an accumulation of life stressors may give rise to AL. In practice, it may be valuable to use a check list like the one proposed by Fava et al. [61]. A high stressor load which taxes the coping resources of the patient with concomitant psychosomatic or psychiatric symptoms is a likely indicator of AL (see table 3 in online suppl. appendix).

\section{Conclusion}

This review has shown that stressors are very important in disease etiology. There is a large scientific literature supporting this notion. Both psychiatric and somatic disorders are affected by long-lasting exposure to adverse stressors. Plausible underlying mechanisms have been studied. Standardized assessments for stressors have also been published and could be widely used clinically. The assessment of work stressors has often been neglected in clinical work. Given the etiological importance that work stressors have according to the literature, this neglect has not been scientifically justified. Methodological work has resulted in several easily administered and extensively tested questionnaires for work environment and life events. In the online supplementary appendix, the reader can find easily administered instruments that are free for use: table 1 , a short questionnaire regarding serious life events; table 2, an interview guide regarding life events, and table 3 , a list of criteria for AL. In addition, the reader may contact web pages which present other instruments which could be used clinically or for research. See for instance questionnaires about life events, coping, social support: www.drrahe.com - a questionnaire regarding working conditions in general, www.mentalhealthpromotion.net/resources/english_copsoq_2_ed_2003-pdf.pdf - a questionnaire specifically designed to measure effort reward imbalance at work, and www.workhealth.org/UCLA\%20OHP\%20class\%202004/ERI\%202004.pdf - a questionnaire designed to assess working conditions in general, particularly psychological demands, decision latitude and social support at work.

\section{References}

$>1$ Brown GW, Sklair F, Harris TO, Birley JL: Life events and psychiatric disorders. 1. Some methodological issues. Psychol Med 1973;3:74-78.

2 Theorell T: Critical life events and cardiovascular disease. In Cooper CL (ed). Handbook of Stress, Medicine, and Health. CRC Press, Boca Raton, 1996, p 33.

3 McEwen BS: Protective and damaging effects of stress mediators. N Engl J Med 1998;338:171-179.

4 Kasl S, Gore S, Cobb S: The experience of losing a job: Reported changes in health, symptoms and illness behavior. Psychosom Med 1975;37:106-122.
5 Arnetz BB, Brenner SO, Levi L, Hjelm R, Petterson IL, Wasserman J, Petrini B, Eneroth P, Kallner A, Kvetnansky R, Vigas M: Neuroendocrine and immunologic effects of unemployment and job insecurity. Psychother Psychosom 1991;55:76-80.

O'Connor M: A longitudinal study of PTSD in the elderly bereaved: prevalence and predictors. Aging Ment Health 2010;14:310-318, erratum in Aging Ment Health 2010;14:iii. 
7 Ironson G, Wynings C, Schneiderman N, Baum A, Rodriguez M, Greenwood D, Benight C, Antoni M, LaPerriere A, Huang HS, Klimas N, Fletcher MA: Posttraumatic stress symptoms, intrusive thoughts, loss, and immune function after Hurricane Andrew. Psychosom Med 1997;59:128-141.

8 Holmes TH, Rahe RH: The social readjustment rating scale. J Psychosom Res 1967;11:213-218.

$\checkmark 9$ Rahe RH: Life changes, stress responsivity, and captivity research. Psychosom Med 1990;52:373-396.

10 Theorell T, Lind E, Fröberg J, Karlsson C-G, Levi L: A longitudinal study of 21 subjects with coronary heart disease: life changes, catecholamines and related biochemical variables. Psychosom Med 1972;34:505-516.

11 Paykel ES: The interview for recent life events. Psychol Med 1997;27:301-310.

12 Miller MA, Rahe RH: Life changed scaling for the 1990s. J Psychosom Res 1997;43:279-292.

$\checkmark 13$ Moller J, Theorell T, de Faire U, Ahlbom A, Hallqvist J: Work related stressful life events and the risk of myocardial infarction. Case-control and case-crossover analyses within the Stockholm heart epidemiology programme (SHEEP). J Epidemiol Community Health 2005;59:23-30.

14 Theorell T, Lind E, Flodérus B: The relationship of disturbing life-changes and emotions to the early development of myocardial infarction and other serious illnesses. Int J Epidemiol 1975;4:281-293.

15 Rafanelli C, Roncuzzi R, Milaneschi Y, Tomba E, Collstro MC, Pancaldi LG, Di Pasquale G: Stressful live events, depression and demoralization as risk factors for acute coronary disease. 2005;74:179_ 184.

16 Maclure M, Mittleman MA: Should we use a casecrossover design? Annu Rev Public Health 2000; 21:193-221.

17 Paykel ES: Life stress, depression and attempted suicide. J Hum Stress 1976;2:3-12.

18 Theorell T, Svensson J, Knox S, Waller D, Alvarez M: Young men with high blood pressure report few recent life events. J Psychosom Res 1986;30:243249.

19 Theorell T, Emlund N: On physiological effects of positive and negative life changes - a longitudinal study. J Psychosom Res 1993;37:653-659.

20 Svensson J, Theorell T: Life events and elevated blood pressure in young men. J Psychosom Res 1983;27:445-455.

21 Dohrenwend BS, Dohrenwend BP (eds): Stressful Life Events, their Nature and Effects. New York, Wiley 1974.

22 Paykel ES: Life events and affective disorders. Acta Psychiatr Scand Suppl 2003;418:61-66.
23 Rahe RH: Paths to Health and Resilience through Stress and Coping Wisdom, 2009. Available for downloading at amazon.com.

24 Brown GW and Harris TO (eds): Life Events and Illness. Unwin Hyman, London 1989.

25 Brown GW: Measurement and the epidemiology of childhood trauma. Semin Clin Neuropsychiatry 2002;7:66-79.

26 Karasek RA, Theorell T: Healthy Work. Basic Books, New York 1990.

27 Schnall PL, Dobson M, Rosskam E (eds): Unhealthy Work: Causes, Consequences, Cures. Baywood Publishing Company, Amityville, NY 2009.

28 Marmot M, Siegrist J, Theorell T: Health and the psychosocial environment at work. In: Social Determinants of Health, second ed. (eds) Marmot $\mathrm{M}$ and Wilkinson RG Oxford University Press Inc., New York 2006, pp 97-130.

29 Levi L, Levi I: Guidance on work-related stress. Spice of life or kiss of death. Luxembourg, Office for Official Publications of the European Communities, 2000.

30 Belkic KL, Landsbergis PA, Schnall PL, Baker D: Is job strain a major source of cardiovascular disease risk? Scand J Work Environ Health 2004;30:85-128.

31 Kivimaki M, Virtanen M, Elovainio M, Kouvonen A, Vaananen A, Vahtera J: Work stress in the etiology of coronary heart disease-a meta-analysis. Scand J Work Environ Health 2006;32:431-442.

32 Eller NH, Netterstrom B, Gyntelberg F, et al: Workrelated psychosocial factors and the development of ischemic heart disease: a systematic review. Cardiol Rev 2009;17:83-97.

33 Gidron Y, Kupper N, Kwaijtal M, Winter J, Denollet $\mathrm{J}$ : Vagus-brain communication in atherosclerosisrelated inflammation: a neuroimmunomodulation perspective of CAD. Atherosclerosis 2006;195: e1-e9.

34 Belkić KL, Schnall PL, Landsbergis PA, Schwartz JE, Gerber LM, Baker D, Pickering TG: Hypertension at the workplace - an occult disease? The need for work site surveillance. Adv Psychosom Med 2001; 22:116-138.

35 Theorell T, Perski A, Åkerstedt T, Sigala F, AhlbergHultén G, Svensson J, Eneroth P: Changes in job strain in relation to changes in physiological state a longitudinal study. Scand J Work Env Health 1988; 14:189-196.

36 Theorell T, Karasek RA, Eneroth P: Job strain variations in relation to plasma testosterone fluctuations in working men - a longitudinal study. J Intern Med 1990a;227:31-36.

37 Theorell T, Orth-Gomér K, Eneroth P: Slowreacting immunoglobulin in relation to social support and changes in job strain. Psychosom Med 1990b;52:511-516. 
38 Collins SM, Karasek RA, Costas K: Job strain autonomic indices of cardiovascular disease risk. Am J Ind Med 2005;48:182-193.

39 Maina G, Bovenzi M, Palmas A, Filon F: Associations between two job stress models and measures of salivary cortisol. Int Arch Occup Environ Health 2009;82:1141-1150.

40 Hansen AM, Larsen AD, Rugulies R, Garde AH, Knudsen LE: A review of the effect of the psychosocial working environment on physiological changes in blood and urine. Basic Clin Pharmacol Toxicol 2009;105:73-83.

41 Theorell T: Democracy at work and its relationship to health. In: Research in Occupational Stress and Well being. Emotional and Physiological Processes and Positive Intervention Strategies. (eds) Perrewé ML and Ganster DC, Amsterdam, Elsevier 2004, vol 3, pp 323-357.

-42 Wang HX, Leineweber C, Kirkeeide R, Svane B, Schenck-Gustafsson K, Theorell T, Orth-Gomér K: Psychosocial stress and atherosclerosis: family and work stress accelerate progression of coronary disease in women: The Stockholm Female Coronary Angiography Study. J Intern Med 2007;26:245-254.

43 Siegrist J: Chronic psychosocial stress at work and risk of depression: evidence from prospective studies. Eur Arch Psychiatry Clin Neurosci 2008;258 (suppl 5): 115-119.

44 Kristensen TS, Hannerz H, Høgh A, Borg V: The Copenhagen Psychosocial Questionnaire - a tool for the assessment and improvement of the psychosocial work environment. Scand J Work Environ Health 2005;31:438-449.

-45 Siegrist J, Starke D, Chandola T, Godin I, Marmot M, Niedhammer I, Peter R: The measurement of effort-reward imbalance at work: European comparisons. Soc Sci Med 2004;58:1483-1499.

-46 Theorell T, Emdad R, Arnetz B, Weingarten AM: Employee effects of an educational program for managers at an insurance company. Psychosom Med 2001;63:724-733.

47 Romanowska J, Larsson G, Eriksson M, Wikström $\mathrm{BM}$, Westerlund $\mathrm{H}$, Theorell $\mathrm{T}$ : Health effects on leaders and co-workers of an art-based leadership development program. Psychother Psychosom 2011; 80:78-87.

48 Sensky T: Chronic embitterment and organisational justice. Psychother Psychosom 2010;79:65-72
49 Janlert U: Unemployment as a disease and diseases of the unemployed. Scand J Work Environ Health. 1997;23(suppl 3):79-83.

50 Tsai JF: Socioeconomic factors outweigh climate in the regional difference of suicide death rate in Taiwan. Psychiatry Res 2010;179:212-216.

51 Mattiasson I, Lindgärde F, Nilsson JA, Theorell T: Threat of unemployment and cardiovascular risk factors: longitudinal study of quality of sleep and serum cholesterol concentrations in men threatened with redundancy. BMJ 1990;8301:461-466.

52 Arnetz BB, Wasserman J, Petrini B, Brenner SO, Levi L, Eneroth P, Salovaara H, Hjelm R, Salovaara $\mathrm{L}$, Theorell $\mathrm{T}$, et al: Immune function in unemployed women. Psychosom Med 1987;49:3-12.

53 Hammarström A, Janlert U: Early unemployment can contribute to adult health problems: results from a longitudinal study of school leavers. J Epidemiol Community Health 2002;56:624-630.

54 Westerlund $\mathrm{H}$, Gustafsson PE, Theorell T, Janlert U, Hammarström A: Social adversity in adolescence increases the physiological vulnerability to job strain in adulthood: a prospective population-based study, submitted.

55 Juster RP, McEwen BS, Lupien SJ: Allostatic load biomarkers of chronic stress and impact on health and cognition. Neurosci Biobehav Rev 2010;35:216.

56 Sapolsky RM, Romero LM, Munck AU: How do glucocorticoids influence stress responses? Integrating permissive, suppressive, stimulatory, and preparative actions. Endocr Rev 2000;21:55-89.

57 Seeman TE, McEwen BS, Rowe JW, et al: Allostatic load as a marker of cumulative biological risk: MacArthur studies of successful aging. Proc Natl Acad Sci USA 2001;98:4770-4775.

58 Seeman TE, Singer BH, Rowe JW, et al: Price of adaptation - allostatic load and its health consequences. MacArthur studies of successful aging. Arch Intern Med 1997;157:2259-2268.

-59 Karlamangla AS, Singer BH, Seeman TE: Reduction in allostatic load in older adults is associated with lower all-cause mortality risk: MacArthur studies of successful aging. Psychosom Med 2006;68:500-507.

60 Chandola T, Brunner E, Marmot M: Chronic stress at work and the metabolic syndrome: prospective study. BMJ 2006;332:521-525.

61 Fava GA, Guidi J, Semprini F, Tomba E, Sonino N: Clinical assessment of allostatic load and clinimetric criteria. Psychother Psychosom 2010;79:280-284.

Prof. Töres Theorell

Institute for Stress Research

Stockholm University

SE-10691 Stockholm (Sweden)

Tel. +46 85537 8925, E-Mail Tores.Theorell@stressforskning.su.se 